Vol. 5(3), pp. 31-38, April 2014

DOI: 10.5897/J YFR2014.0134

Artic le Number: 174824644316

ISSN 2141-2413

Copyright (c) 2014

Author(s) reta in the copyright of this artic le

http://www.academic joumals.org/J YFR

\title{
Effect of co-culturing of cellulolytic fungal isolates for degradation of lignocellulosic material
}

\author{
K. Mohanan ${ }^{1}$, R.R. Ratnayake ${ }^{1 *}$, K. Mathaniga ${ }^{2}$, C. L. Abayasekara ${ }^{3}$ and N. Gnanavelrajah ${ }^{2}$ \\ ${ }^{1}$ Institute of Fundamental Studies, Hantana Road, Kandy, Sri Lanka. \\ ${ }^{2}$ Faculty of Agriculture, University of Jaffna, Sri Lanka. \\ ${ }^{3}$ Department of Botany, University of Peradeniya, Peradeniya, Sri Lanka.
}

Received 10 March, 2014; Accepted 10 April, 2014

\begin{abstract}
This study intended to compare the efficiency of fungal monocultures and co-cultures in the simultaneous delignification and saccharification of kitchen waste and Eichhornia crassipes in order to subject the hydrolysate into biofuel production. Three fungal isolates of genus Trichoderma, Aspergillus, Pycnoporus and an unidentified strain (F113) were grown in mono and co-cultures and the extracted enzymes were used for the degradation. Co-culture of Trichoderma spp with the other fungi improved its enzyme activity while the other co-cultures did not show significantly improved enzymatic degradation compared to monocultures. The highest percentage of saccharification (over total dry weight) achieved were $11.9 \%$ with kitchen waste after seven days and $9.8 \%$ with $E$. crassipes after 4 days. The drop in degradation rate normally seen after complete digestion of amorphous cellulose was not apparent probably due to the grinding of the substrates to fine particle size.
\end{abstract}

Key words: Fungal co-culture, biofuel, cellulase, kitchen waste, invasive weeds.

\section{INTRODUCTION}

Fossil fuel resources are limited and their usage leads to environmental problems. Hence, it is imperative to utilise alternative energy sources that are renewable and ecofriendly. Biofuels are promising in this regard. Second generation biofuels are produced from non-edible biomass, through degradation and fermentation.

Enzymes degrading lignocelluloses in nature include cellulases, xylanases and lignin degrading enzymes. The activity of lignin degrading enzyme is too slow for application in biofuel production (Lu et al., 2010). Thus, thermo-chemical pre-treatment is needed to overcome the recalcitrance (Margeot et al., 2009). Pre-treatment also results in degradation of hemicelluloses. Therefore, cellulases are the major enzymes involved in subsequent enzymatic hydrolysis.

Pre-treatment requires energy input and chemicals which adds up to a major component of the cost of production. The cost of enzymes also contributes significantly (Shi et al., 2009). Among microbes, some aerobic filamentous fungi are known to secrete high

${ }^{*}$ Corresponding author. E-mail: renukar@ifs.ac.Ik. Tel: + 94-81-2232002. Fax: + 94-81-2232131.

Author(s) agree that this article remain permanently open access under the terms of the Creative Commons Attribution License 4.0 International License 
amounts of cellulases. Such strains are found in the genera Trichoderma, Aspergillus, Pencillium and many others (Lynd et al., 2002). The factors affecting the expression and secretion of fungal cellulases include induction, repression, metal ions, inorganic nutrients, surfactants and culture conditions (Gremel et al., 2008; Mandels and Reese, 1957; Reese and Maguire, 1969; Schmoll et al., 2005; Suto and Tomita, 2001). Cellulases belong to three classes, namely endoglucanases, exoglucanases and $\beta$-glucosidases, which act in synergy (Lynd et al., 2002). The right balance of the cellulases is crucial for optimal degradation of lignocelluloses. In Trichoderma reesei, large proportions of $\beta$-glucosidase remain cell-wall bound through a polysaccharide and gets released when treated with cellulase from Aspergillus niger (Messner et al., 1990). Endoglucanases and exoglucanases of $T$. reesei are inhibited by cellobiose whereas its $\beta$-glucosidases was inhibited by glucose (Philippidis et al., 1993). In contrast, A. niger secretes high amounts of $\beta$-glucosidase which is tolerant to high levels of glucose (Decker et al., 2000). For these reasons, a combination of cellulases from $T$. reesei and $A$. niger is used in the biofuel industry (Reczey et al., 1998). Co-culture of $T$. reesei and Aspergillus sp has been shown to result in better yield of cellulases (Ahamed and Vermette, 2008; Duff, 1985). Better utilization of substrates and formation of strong inducers for $T$. reesei cellulases by $\beta$-glucosidase of Aspergillus are thought to be the reasons for the increased yield (Ahamed and Vermette, 2008).

Disposal of household waste is a problem in the urban areas of Sri Lanka. Current practice is to dump the waste in the suburbs, causing unpleasant odours and health problems in those areas. Composting has been attempted in an industrial scale but has been abandoned due to operational problems (Premachandra, 2006). A possible solution to this problem could be the utilization of household waste for biofuel production. Another potential substrate is the invasive aquatic weed Eichhornea crassipes. Invasive weeds are probable raw materials for cellulosic biofuel production. These are non-indigenous or "non-native" plants which adversely affect the habitats and bioregions they invade economically, environmentally, and ecologically (Westbrooks, 1998). E. crassipes is one of a common aquatic invasive weed and another potential substrate for biofuel production. The fast growing nature of these weeds can provide raw materials in abundance for biofuel industries. Since it is less lignified, its utilisation would require low pretreatment. If effective technologies can be developed to drive commercial products from these weeds it will be beneficial both economically and ecologically. Although a large number of microorganisms (fungi, bacteria and actinomycetes) are capable of degrading cellulose, only a few of them produce significant quantities of cell-free enzyme fractions capable of complete hydrolysis of cellulose in vitro. Cellulases obtained from compatible mixed cultures of fungi appear to have more enzyme activity as compared to their pure cultures and other combinations (Jayant et al., 2011).

The objective of this study was to compare the efficiency of fungal monocultures and co- cultures in the simultaneous delignification and saccharification of kitchen waste and E. crassipes in order to subject the hydrolysate into biofuel production.

\section{MATERIALS AND METHODS}

\section{Isolation of cellulolytic fungi}

Samples of decaying plant material, ruminant dung, decaying kitchen waste and soil were suspended in sterile normal saline, serially diluted to obtain $10^{-1}, 10^{-2}$ and $10^{-3}$ dilutions. $100 \mu \mathrm{l}$ of each dilution was plated on potato dextrose agar (with gentamicin 50 $\mathrm{mg} / \mathrm{L}$ and chloramphenicol $50 \mathrm{mg} / \mathrm{L}$ ) by spread plate technique and incubated at $25^{\circ} \mathrm{C}$ up to a week. The fungal isolates were inoculated on Czapek dox agar without sucrose and with $1 \%$ cellulose and those showing good growth were presumed to be potential cellulose degraders and added to the culture collection. The isolates in the culture collection were screened for cellulase production by growing them without replicates as described in measurement of enzyme activities part of the work. The isolates which showed at least 0.01 FPU of cellulase activity were then tested in replicates and those with the highest enzyme activities from different genera were chosen for further study.

\section{Measurement of enzyme activities}

\section{Production of enzymes}

Fungal isolates were grown on PDA slants for 7 days. Spore suspensions were made in sterile saline, spore concentrations were adjusted to $10^{7}-10^{8} / \mathrm{ml}$ and $100 \mu \mathrm{l}$ of the suspensions were inoculated into $20 \mathrm{ml}$ of a minimal medium (Mandels and Reese, 1957). Co-cultures were made by inoculating $100 \mu \mathrm{l}$ of spore suspension from each of the relevant strain. The cultures were incubated at $28^{\circ} \mathrm{C}$ on a rotary shaker at $100 \mathrm{rpm}$. For initial screening, the isolates were cultured without replicates with an incubation period of 3 days. At the end of incubation, the cultures were centrifuged at $4000 \mathrm{~g}$ for $20 \mathrm{~min}$ and the supernatants were used as crude enzymes for the assays.

\section{Total cellulase assay}

Total cellulase assay was carried out using Whatmann No.1 filter paper as the substrate (Mandels et al., 1976; Ghose, 1987). Reducing sugars formed were measured by using di-nitro salicylic acid reagent (Sumner, 1921; Miller, 1959), with glucose as standard. The total cellulase activity is expressed as filter paper units/ml (FPU/ml).

\section{Xylanase assay}

Xylanase activities were measured by a method modified from Gottschalk et al. (2010) using 1\% (w/v) beech wood xylan (Sigma) as the substrate. Reducing sugars formed were measured using dinitro salicylic acid (DNS) reagent (Sumner, 1921; Miller, 1959), with xylose as standard. 


\section{$\beta$-Glucosidase assay}

$\beta$-glucosidase activities were measured by using cellobiose as the substrate (Ghose, 1987; Sternberg et al., 1977). Glucose formed during the assay was measured using a commercial blood glucose meter (One Touch Ultra 2) based on glucose oxidase, calibrated with glucose standards in $0.05 \mathrm{M}$ citrate buffer $(\mathrm{pH}=4.8)$. Positive results of $\beta$-glucosidase activities were verified by high-performance liquid chromatography (HPLC) as described in enzymatic degradation of lignocellulosic materials part of this work.

\section{Laccase assay}

Laccase activities of Pycnoporus cinnabarinus was measured with ABTS (2,2'-azino-bis(3-ethylbenzothiazoline- 6-sulphonic acid)) as the substrate (Bourbonnais et al., 1995).

\section{Enzymatic degradation of lignocellulosic materials}

Kitchen waste and mature leaves (to represent the most recalcitrant type for degradation, as they are the most lignified) of $E$. crassipes were dried in an oven at $50^{\circ} \mathrm{C}$ to a constant weight. The dried material was then ground in a plant grinder and sieved through 93 $\mu \mathrm{m}$ (kitchen waste) and $50 \mu \mathrm{m}$ (E. crassipes) sieve. Powdered kitchen waste $(500 \mathrm{mg})$ and E. crassipes $(200 \mathrm{mg})$ were added separately into boiling tubes, to which $6 \mathrm{ml}$ of $50 \mathrm{mM}$ citrate buffer $\left(\mathrm{pH} \mathrm{4.8)}\right.$ was added and autoclaved at $121^{\circ} \mathrm{C}$ for $15 \mathrm{~min}$. To each tube, $3 \mathrm{ml}$ of crude enzyme from fungal monoculture or co-culture was added and incubated in a water bath at $50^{\circ} \mathrm{C}$ with reciprocal shaking at $100 \mathrm{rpm}$ for five to seven days. Samples were withdrawn daily and total sugar concentrations were determined using DNS reagent with glucose standards (Sumner et al., 1921). The sugar components of the hydrolysate of $E$. crassipes at the end of 4 th day of degradation were measured by High-performance liquid chromatography (HPLC) using agilent Hi-plex $H$ column ( $p / n$ PL1170-6830 $300 \times 7.7 \mathrm{~mm})$ at $65^{\circ} \mathrm{C}$ with deionised water as the mobile phase (flow rate: $0.6 \mathrm{ml} / \mathrm{min}$, injection volume: $10 \mu \mathrm{l}$ ). Sugars were detected with an RI detector. D-cellobiose, D-glucose, Dxylose and L-arabinose (Sigma Aldrich) standards were used for calibration. High-performance liquid chromatography was not performed on kitchen waste due to the highly variable nature of its content.

\section{Data analysis}

Statistical comparisons were made by ANOVA using Minitab software (version 14). $\alpha=0.05$ unless otherwise stated.

\section{RESULTS AND DISCUSSION}

\section{Isolation of cellulolytic fungi}

A total of 145 fungal strains were isolated from different samples. During the initial screening for cellulase activity, 35 isolates were found to have greater than $0.01 \mathrm{FPU} / \mathrm{ml}$ of activity. The isolates which showed significant cellulase activities were shown in Table 1. Most of them belong to Trichoderma species, while strains of Penicillium, Aspergillus and an unidentified fungal strain were also present. Fungi were tentatively identified using macroscopic and microscopic morphological characteris- tics. Slide culture technique was used to aid the fungal identifications.

\section{Co-culture of fungi}

Fungal isolates belonging to genera of Trichoderma ( $\mathrm{F} 1$, F16,F F118), Penicillium (F24) and an unidentified isolate (F113) were selected for co-culturing. The selection was made to include different genera and strains with $\beta$ glucosidase activity. The isolates were divided into two groups (F1, F16 and F118) and (F24, F80, F113) and cocultures were made in all possible combinations within each group. Cellulase and xylanase activities of the cocultures and corresponding mono-cultures were measured (Figures 1 and 2).

Among the group containing F1, F16 and F118, the cocultures showed lower cellulase activities compared to the corresponding monocultures. Among the group containing F24, F80 and F113, the co-culture F80 and F113 showed higher cellulase activity than F113, but the difference was statistically insignificant. The co-cultures F24/F113 and F24/F80/F113 showed significantly higher xylanase activities compared to the corresponding monocultures. Other co-cultures showed either no significant difference or reduced xylanase activity.

\section{Degradation of kitchen waste}

The isolates F24, F113 and F118 were selected for degradation of kitchen waste. The cumulative sugar contents measured at $1^{\text {st }}, 2^{\text {nd }} 3^{\text {rd }}$ and $7^{\text {th }}$ days of degradation of fresh kitchen waste was shown in Figure 3 . At the end of seven days of degradation, the highest amount of sugars were released by enzymes from F24 $(59.7 \mathrm{mg})$ followed by the co-culture F24/F113 $(58 \mathrm{mg})$. Enzymes from the co-culture F113/F118 effected significantly higher degradation than F118 (Trichoderma) alone. This is probably due to the lack of secreted $\beta$ glucosidase activity by F118 being complemented by F113.

\section{Degradation of $E$. crassipes}

Enzymes from the fungal strains F24, F113, F118 and a woody mushroom from Sri Lanka (M21), identified as Pycnoporus cinnabarinus, were used for the degradation of $E$. crassipes. Screening revealed that some organisms were more efficient than the others. M21 was found to have a significant cellulase activity (0.21 FPU) and laccase activity $(50 \mathrm{IU} / \mathrm{ml})$. The cumulative sugar contents measured at $1^{\text {st }}, 2^{\text {nd }}, 3^{\text {rd }}$ and $4^{\text {th }}$ days of enzymatic degradation of $E$. crassipes was shown in Figure 4. The sugar contents at the $4^{\text {th }}$ day was shown in Figure 5 for comparison. The component sugars as 
Table 1. Fungal isolates with highest cellulase activities.

\begin{tabular}{ccccc}
\hline Isolate no. & $\begin{array}{c}\text { Total cellulase } \\
(\text { FPU/mI) }\end{array}$ & $\begin{array}{c}\text { Xylanase } \\
(\mathbf{I U} / \mathbf{m l})\end{array}$ & $\begin{array}{c}\boldsymbol{\beta} \text {-glucosidase } \\
(\mathbf{I U} / \mathrm{ml})\end{array}$ & Genus \\
\hline F118 & 0.21 & 4.31 & Not detected & Trichoderma \\
F80 & 0.16 & 2.03 & Not detected & Trichoderma \\
F1 & 0.15 & 5.48 & Not detected & Trichoderma \\
F16 & 0.14 & 5.22 & 0.11 & Trichoderma \\
F22 & 0.13 & 5.22 & 0.09 & Trichoderma \\
F24 & 0.12 & 4.98 & Not detected & Penicillium \\
F54 & 0.11 & 1.77 & Not detected & Trichoderma \\
F27 & 0.11 & 5.38 & Not detected & Trichoderma \\
F98 & 0.10 & 4.79 & 0.05 & Trichoderma \\
F10 & 0.09 & 2.14 & Not detected & Trichoderma \\
F56 & 0.08 & 5.62 & 0.05 & Trichoderma \\
F88 & 0.07 & 5.25 & 0.13 & Trichoderma \\
F40 & 0.06 & 3.35 & 0.07 & Aspergillus \\
F113 & 0.06 & 1.02 & 0.15 & Unidentified \\
\hline
\end{tabular}

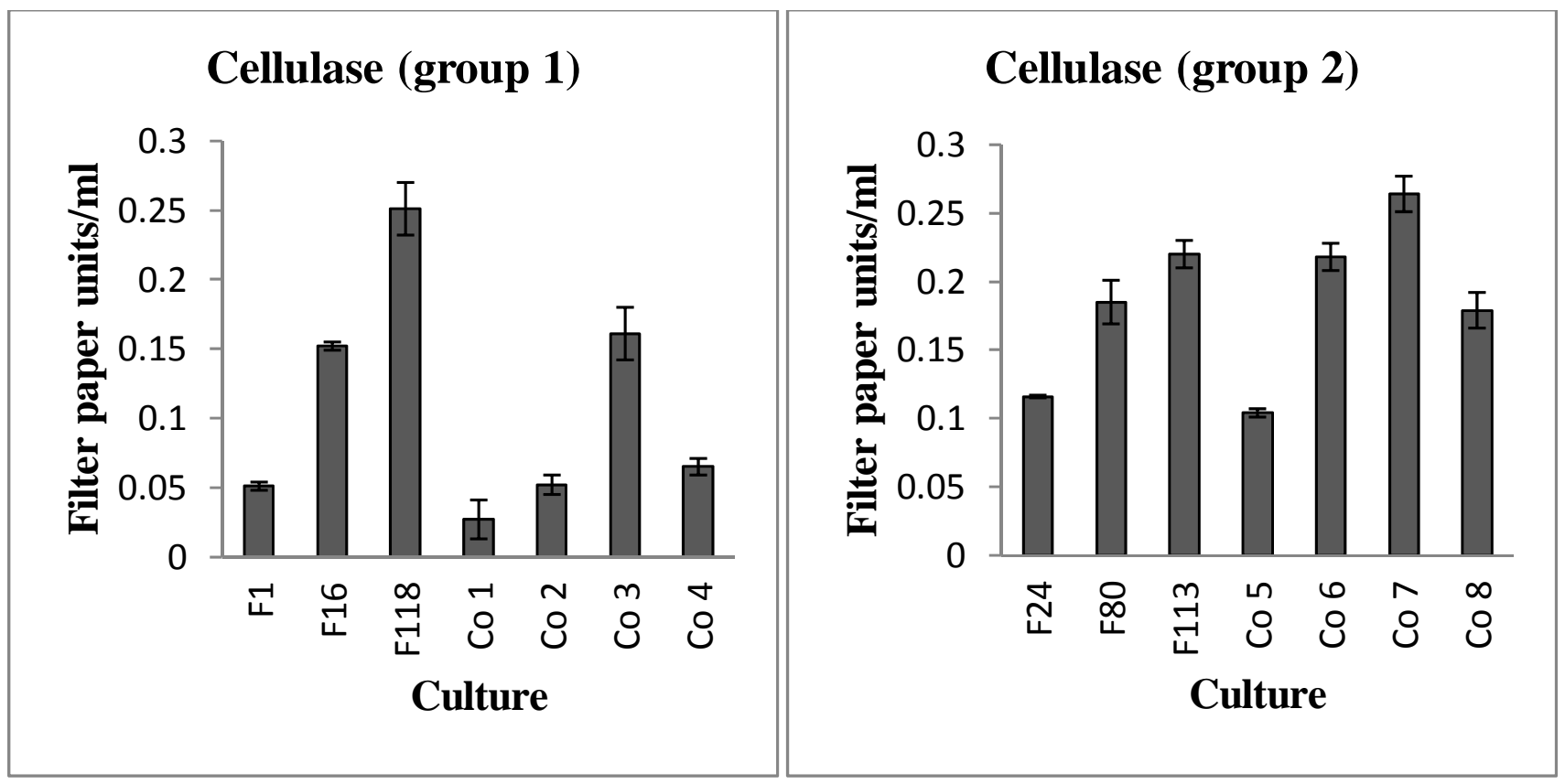

Figure 1. Cellulase activities of monocultures and co-cultures of selected fungi. Key: F1-Trichoderma spp., F16 - Trichoderma spp., F118-Trichoderma spp., Co1- F1 and F16, Co2-F1 and F118, Co3- F16 and F118, Co4- F1, F16 and F118, F24-Penicillium spp., F80-Trichoderma spp., F113-Unidentified, Co5- F24 and F80, Co6- F24 and F113, Co7- F80 and F113, Co8- F24, F80 and F113. Error bars indicate standard errors of the means.

measured by HPLC at the end of $4^{\text {th }}$ day are shown in Figure 6. The amount of simple sugar produced and released to the medium can depend on activities of cellulase systems and physiological characteristics of particular species.

The highest quantity of total sugar content $(19.6 \mathrm{mg})$ at the end of four days of degradation of $E$. crassipes was obtained with enzymes from the co-culture F113/F118. However, the mono-cultures F24, F113 and co-cultures F24/F113, F24/F118 and F24/M21 gave slightly lower quantities and the differences are not statistically significant. F118 and F113/M21 and M21 showed significantly lower degradation rate. It was noted that while the amount of xylose formed by M21 was similar to 

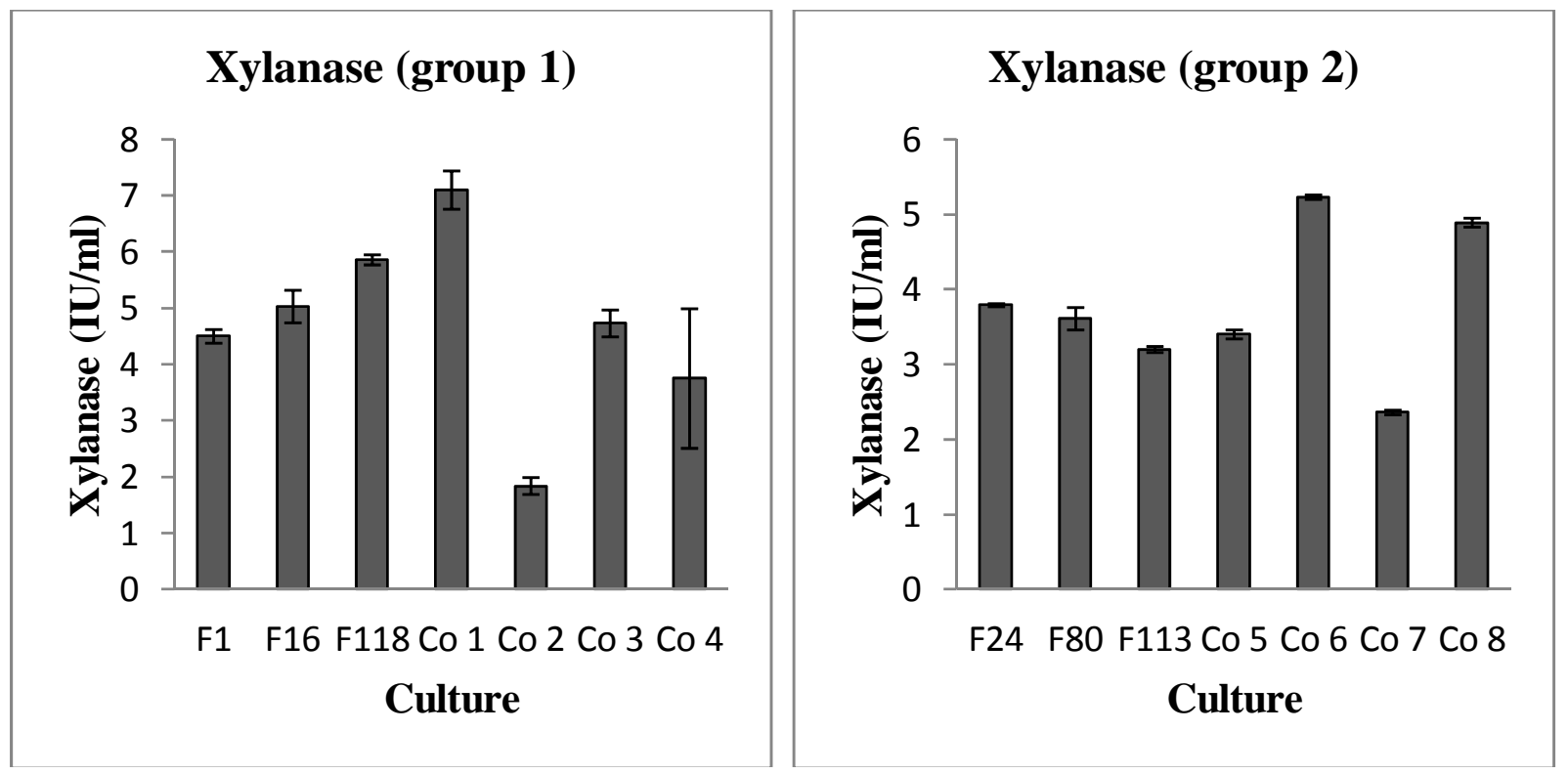

Figure 2. Xylanase activities of monocultures and co-cultures of selected fungi. Key: F1-Trichoderma spp., F16 Trichoderma spp., F118-Trichoderma spp., Co1- F1 and F16, Co2-F1 and F118, Co3- F16 and F118, Co4- F1, F16 and F118, F24-Penicillium spp., F80-Trichoderma spp., F113-Unidentified, Co5- F24 and F80, Co6- F24 and F113, Co7- F80 and F113, Co8- F24, F80 and F113. Error bars indicate standard errors of the means.

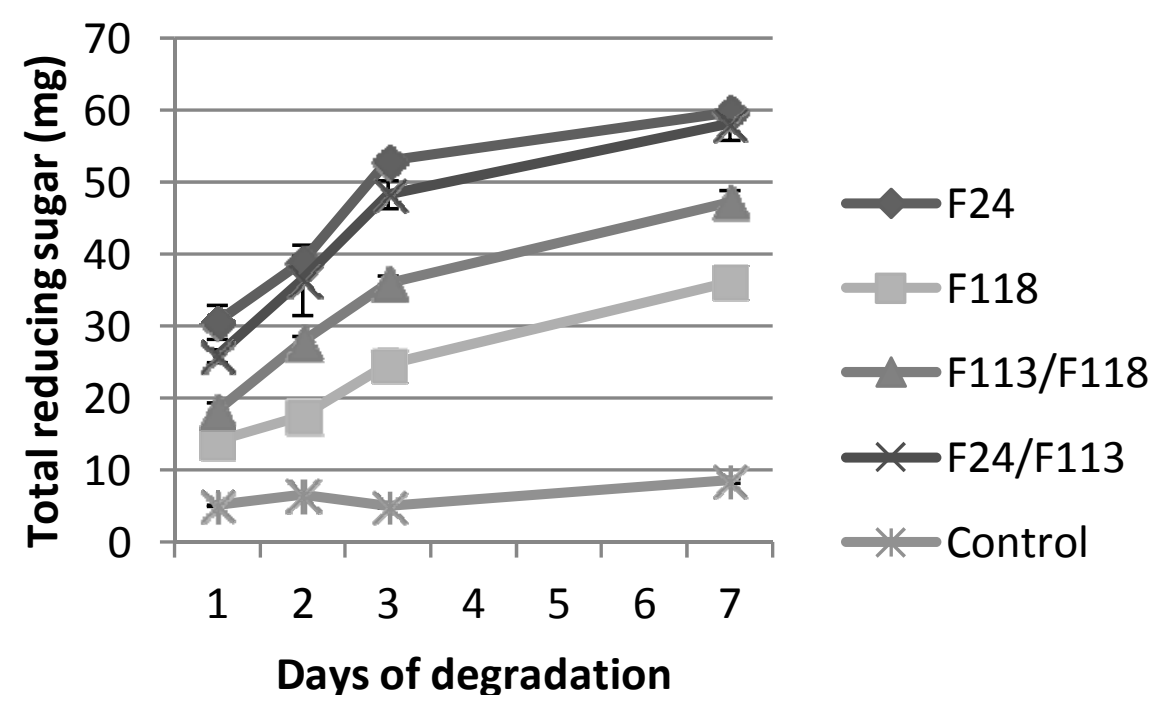

Figure 3. Total sugar content (cumulative) released from un-decomposed kitchen waste by enzymes

other isolates, the amount of arabinose formed was much lower. The percentage of maximum sugar yield over total dry weight was 11.9 and $9.8 \%$ respectively from kitchen waste and $E$. crassipes. The percentages against total polysaccharide content should be higher.

Grinding the substrate to a very fine powder (93 and 50 $\mu \mathrm{m})$ would reduce the length of the polysaccharide chains and increase the number of free ends available for the activity of exoglucanases. It would also increase the surface area of the substrate available for enzyme activity. During the enzymatic hydrolysis of cellulose, amorphous portions are quickly degraded followed by slow degradation of crystalline regions (Mandels, 1975). Thus a change in the rate of degradation, that is, slope of the degradation curve, indicates the end of degradation of amorphous portions. This change should occur roughly 


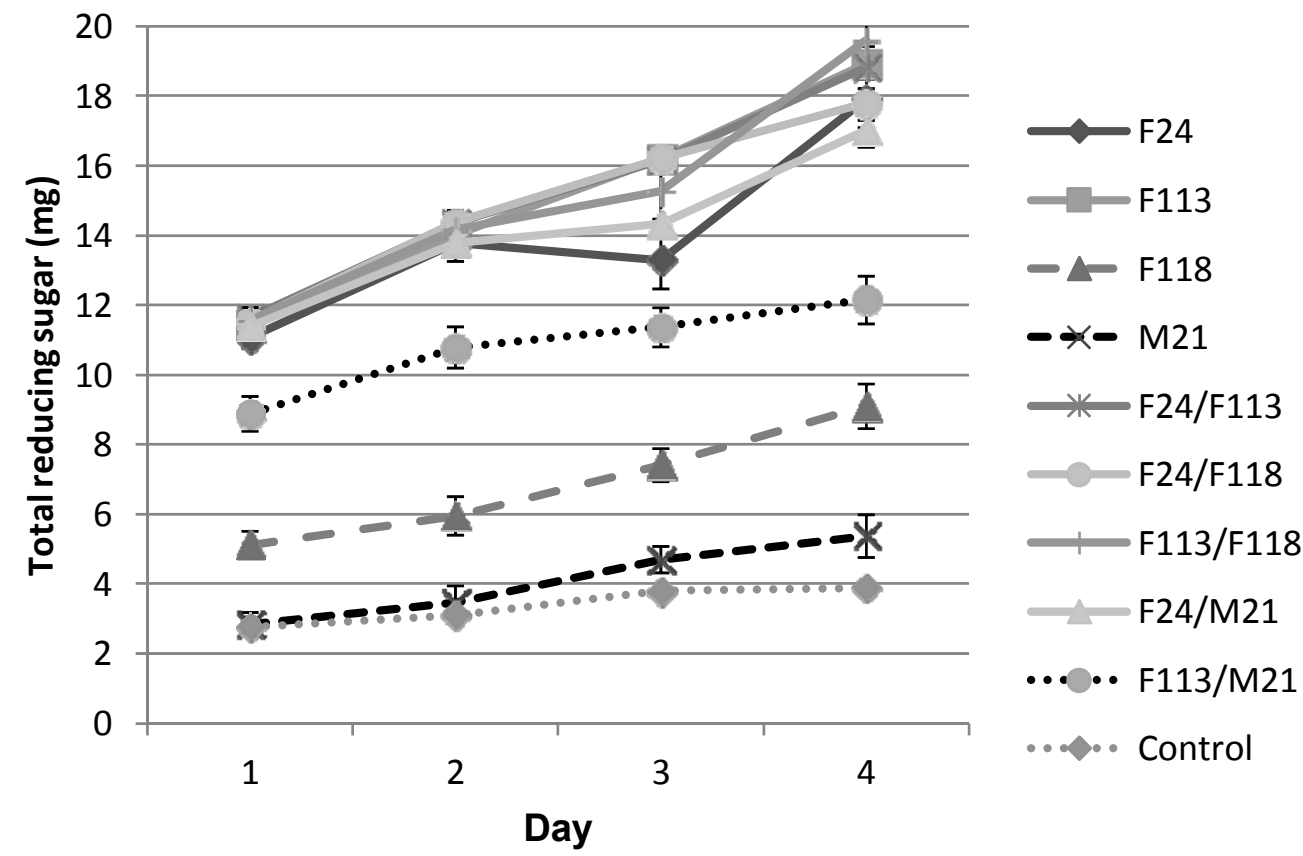

Figure 4. Total reducing sugars measured at daily intervals during enzymatic degradation of $E$. crassipes

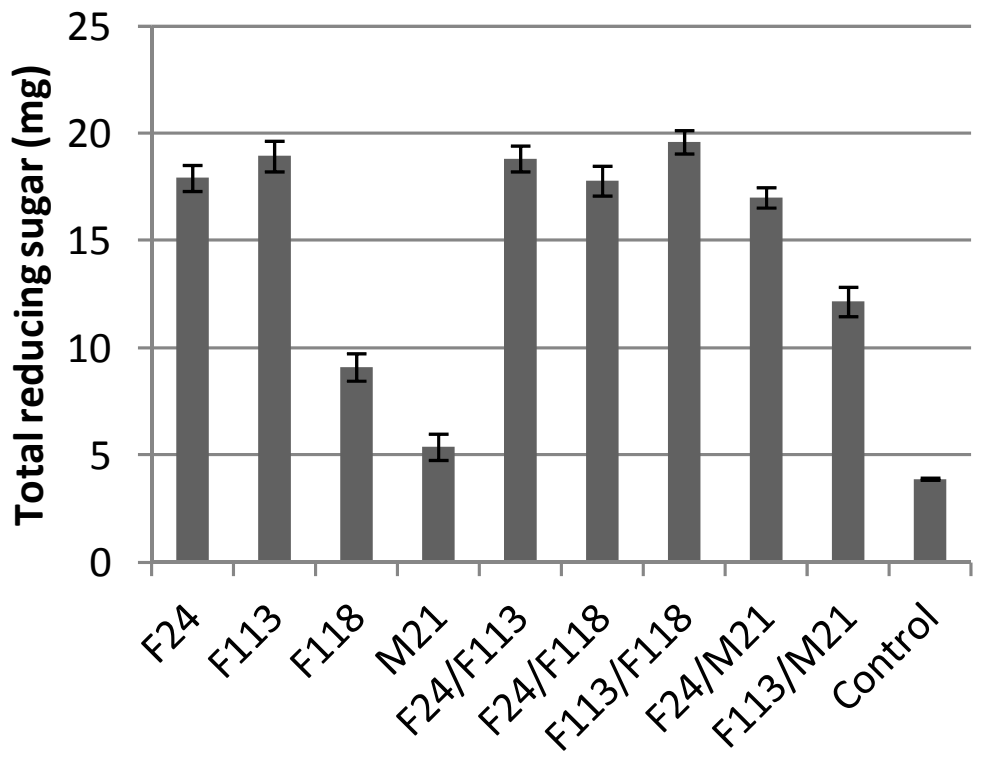

\section{Culture}

Figure 5. Total reducing sugar accumulated by degradation of $E$. crassipes by the end of $4^{\text {th }}$ day of degradation

at about the same percentage of degradation for different enzymes. However, in the present study, such change of rate did not seem to occur at roughly equal percentage of degradation. This could be because at low particle sizes, the effect of enzyme loading becomes more prominent.

\section{Conclusions}

Co-culturing of Trichoderma with other cellulolytic fungi improved the activity of lignocellulose degrading enzymes compared to monoculture of Trichoderma. The co-culture 


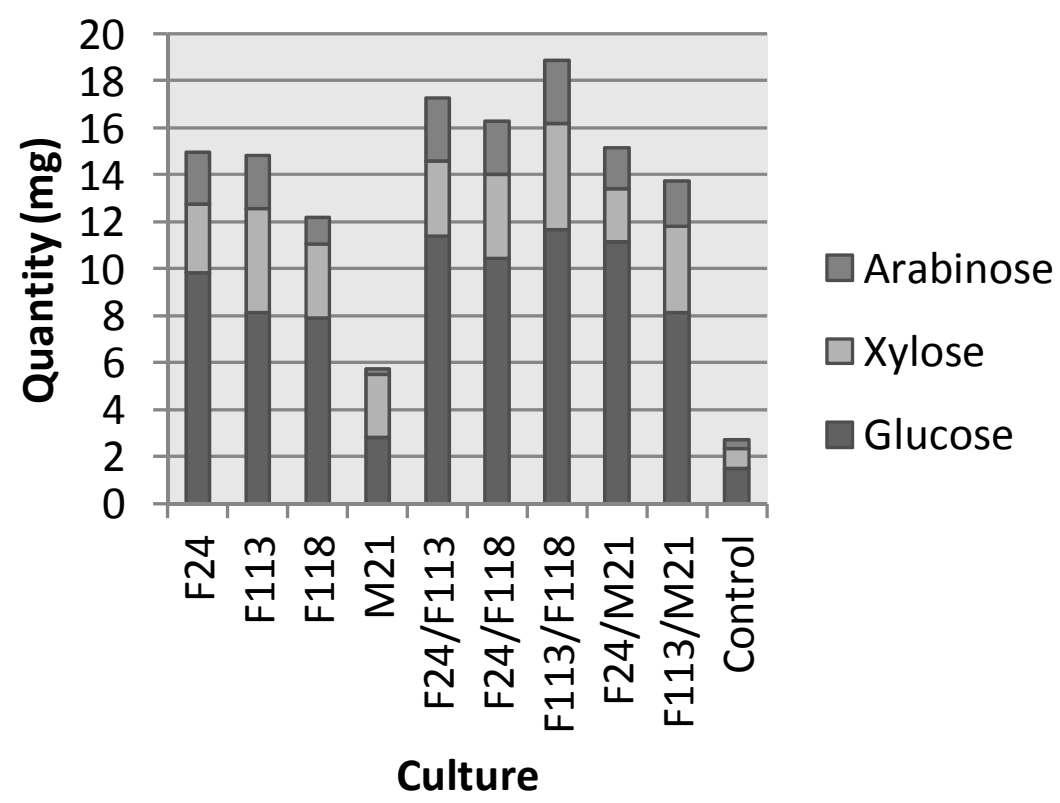

Figure 6. Quantities of glucose, xylose and arabinose of the enzyme hydrolysate of $E$. crassipes at the end of $4^{\text {th }}$ day of degradation.

of other fungi did not result in significant improvement in the activity compared to corresponding monocultures. Significant percentage of degradation of kitchen waste and $E$. crassipes was achieved with the monoculture, without pre-treatment. Fine powdered material was used to eliminate the cost of pre-treatment. Enzyme from Pycnoporus cinnabarinus the laccase producing strain was found to be ineffective for lignocellulose degradation.

\section{REFERENCES}

Ahamed A, Vermette P (2008). Enhanced enzyme production from mixed cultures of Trichoderma reesei RUT-C30 and Aspergillus niger LMA grown as fed batch in a stirred tank bioreactor. Biochem. Eng. J. 42: 41-46.

Bourbonnais R, Paice MG, Reid ID, Lanthier P, Yaguchi M. (1995). Lignin oxidation by laccase isozymes from Trametes versicolor and role of the mediator 2,2'-azinobis (3-ethylbenzthiazoline-6-sulfonate) in kraft lignin depolymerization. Appl. Environ. Microbiol. 61: 18761880.

Decker CH, Visser J, Schreier P (2000). $\beta$-Glucosidases from five black Aspergillus species: study of their physico-chemical and biocatalytic properties. J. Agr. Food Cchem. 48: 4929-4936.

Duff SJ, Cooper DG, Fuller OM (1985). Cellulase and beta-glucosidase production by mixed culture of Trichoderma reesei Rut C30 and Aspergillus phoenicis. Biotechnol. Lett. 7:185-190.

Ghose T (1987). Measurement of cellulase activities. Pure Appl. Chem. $59: 257-268$

Gottschalk LMF, Oliveira RA, Bon EPS (2010). Cellulases, xylanases, $\beta$-glucosidase and ferulic acid esterase produced by Trichoderma and Aspergillus act synergistically in the hydrolysis of sugarcane bagasse. Biochem. Eng. J. 51: 72-78.

Gremel G, Dorrer M, Schmoll M (2008). Sulphur metabolism and cellulase gene expression are connected processes in the filamentous fungus Hypocrea jecorina (anamorph Trichoderma reesei). BMC Mmicrobiol. 8: 174.

Jayant M, Rashmi J, Shailendra M, Deepesh Y (2011). Production of cellulase by different co-culture of Aspergillus niger and Penicillium chrysogenum from waste paper, cotton waste and baggase. J. Yeast Fungal. Res. 2:24-27.

Lu C, Wang H, Luo Y, Guo L (2010). An efficient system for predelignification of gramineous biofuel feedstock in vitro: Application of a laccase from Pycnoporus sanguineus H275. Process Biochem. 45:1141-1147.

Lynd LR, Weimer PJ, van Zyl WH, Pretorius IS (2002). Microbial cellulose utilization: fundamentals and biotechnology. Microbiol. Mol. Biol. R. 66:506 - 577.

Mandels M. (1975). Microbial sources of cellulase. In Biotechnol. Bioeng. Symp. 5, pp. 81-105.

Mandels M, Andreotti R, Roche C (1976). Measurement of saccharifying cellulase. Biotechnology and Bioengineering Symposium (6)21 - 33.

Mandels M, Reese ET (1957). Induction of cellulase in Trichoderma viride as influenced by carbon sources and metals. J. Bacteriol. 73:269.

Margeot A, Hahn-Hagerdal B, Edlund M, Slade R, Monot $F$ (2009)New improvements for lignocellulosic ethanol. Curr. Opin. Biotech. 20:372 - 380

Messner R, Hagspiel K, Kubicek C P (1990). Isolation of a $\beta$ glucosidase binding and activating polysaccharide from cell walls of Trichoderma reesei. Arch. Microbiol. 154:150-155.

Miller GL (1959). Use of Dinitrosalicylic acid reagent for determination of reducing sugar. Anal. Chem. 31:426 - 428.

Philippidis GP, Smith TK, Wyman C E (1993). Study of the enzymatic hydrolysis of cellulose for production of fuel ethanol by the simultaneous saccharification and fermentation process. Biotechnol. Bioeng. 41: 846-853.

Premachandra HS (2006). Household Waste Composting \& MSW Recycling in Sri Lanka. In Asia $3 R$ conference, Tokyo (available from: www.env.go.jp/recycle/3r/en/asia/02_03-3/08.pdf [Accessed 14 December 2013].

Reczey K, Brumbauer A, Bollok M, Szengyel Z, Zacchi G (1998). Use of hemicellulose hydrolysate for $\beta$-glucosidase fermentation. In Biotechnology for Fuels and Chemicals (pp. 225-235). Humana Press.

Reese ET, Maguire A (1969). Surfactants as stimulants of enzyme production by microorganisms. Appl. Microbiol. 17:242-245.

Schmoll M, Franchi L, Kubicek C P (2005). Envoy, a PAS/LOV domain protein of Hypocrea jecorina (Anamorph Trichoderma reesei), modulates cellulase gene transcription in response to light. 
Eukaryotic cell 4:1998-2007.

Shi J, Ebrik M, Yang B, Wyman CE (2009). The potential of cellulosic ethanol production from municipal solid waste: a technical and economic evaluation. (available from: http://escholarship.org/uc/item/99k818c4 [Accessed 15 December 2013].

Sternberg D, Vuayakumar P, Reese ET. (1977). ß-Glucosidase: microbial production and effect on enzymatic hydrolysis of cellulose. Can. J. Microbiol. 23: 139-147.

Sumner JB (1921). Dinitrosalicylic acid: a reagent for the estimation of sugar in normal and diabetic urine. J. Biol. Chem. 47: 5-9
Suto M, Tomita F (2001). Induction and catabolite repression mechanisms of cellulase in fungi. J. Biosci. Bioeng. 92:305-311.

Westbrooks R. (1998). Invasive plants, changing the landscape of America: Fact book. Federal Interagency Committee for the Management of Noxious and Exotic Weeds (FICMNEW) Washington, D.C. 03 pp. Available at http://digitalcommons.usu.edu/cgi/viewcontent.cgi?article=1489\&cont ext=govdocs 